Chronic Obstructive Pulmonary Diseases: Journal of the COPD Foundation

\title{
Review
}

\section{Anti-cytokines as a Strategy in Alpha-1 Antitrypsin Deficiency}

\author{
Oisín F. McElvaney, MB, MRCPI ${ }^{1}$ Mark P. Murphy, PhD ${ }^{1}$ Emer P. Reeves, PhD $^{1}$ Noel G. McElvaney, MD, DSc ${ }^{1}$
}

\begin{abstract}
For many years, the lung disease associated with alpha-1 antitrypsin (AAT) deficiency (AATD) was perceived as being secondary to an imbalance between this serine protease inhibitor and the target protease, neutrophil elastase (NE). More recently, a greater understanding of the pathways leading to lung inflammation has shed light on new potential attributes and presented AATD as an inflammatory condition in which proteases and neutrophils still play a major role, but in which pro-inflammatory cytokines, either induced by the actions of NE or by other pro-inflammatory processes normally modulated by AAT, are involved. In this review, we will look at the various cytokines centrally involved in AATD lung disease, and how a greater understanding of their contribution may help development of targeted therapies.
\end{abstract}

\begin{abstract}
Abbreviations: alpha-1 antitrypsin, AAT; alpha-1 antitrypsin deficiency, AATD; neutrophil elastase, NE; lung epithelial lining fluid, ELF; bronchoalveolar lavage, BAL; interleukin-8, IL-8; cystic fibrosis, CF; secretory leukoprotease inhibitor, SLPI; phenylmethanesulfonyl fluoride, PMSF; leukotriene B4, LTB4; epithelial-derived neutrophil-activating protein-78, ENA-78; growth-regulated oncogene, GRO; interleukin-6, IL-6; oncostatin-M, OSM; granulocyte-macrophage colony-stimulating factor, GMCSF; vascular endothelial growth factor, VEGF; mitogen-activated protein kinase, MAP-kinase; Toll-like receptor, TLR; nuclear factor kappa-light-chain-enhancer of activated B cells, NF-kB; protease-activated receptor2, PAR2; transforming growth factor alpha, TGFa; epidermal growth factor receptor, EGFR; tumor necrosis factor, TNF; interferen, IFN; extracellular matrix, ECM; soluble immune complex, sIC; N-formyl-L-methionyl-Lleucyl-phenylalanine, fMLP; P-selectin glycoprotein ligand 1, PSGL-1; Matrix-metalloprotease-9, MMP-9; interleukin-1 $\beta$, Il- $\boldsymbol{\beta}$; ligand lipopolysaccharide, LPS; epithelial sodium channel, ENaC; short palate lung and nasal epithelial clone 1, SPLUNC1; interleukin-1 receptor antagonist, IL-1Ra

Funding Support: The writing of this manuscript has been supported by funding from the Alpha-1 Foundation and the Health Research Board of Ireland

Date of Acceptance: December 24, 2019

Citation: McElvaney OF, Murphy MP, Reeves EP, McElvaney NG. Anti-cytokines as a strategy in alpha-1 antitrypsin deficiency. Chronic Obstr Pulm Dis. 2020;7(3):203-213. doi: https://doi.org/10.15326/jcopdf.7.3.2019.0171
\end{abstract}

1 Irish Centre for Genetic Lung Disease, Department of Medicine, Royal College of Surgeons in Ireland, Education and Research Centre, Beaumont Hospital, Dublin, Ireland

\section{Address correspondence to:}

Emer P. Reeves, PhD

Irish Centre for Genetic Lung Disease

Royal College of Surgeons in Ireland

Education and Research Centre

Beaumont Hospital

Dublin 9, Ireland

Email: emerreeves@rcsi.ie

Phone: +35318093877

\section{Keywords:}

alpha-1 antitrypsin; neutrophils; inflammation; cytokines; interleukin-8

\section{Introduction}

Alpha-1 antitrypsin (AAT) deficiency (AATD) is a genetic disorder that classically presents with lung and liver disease. Once considered a rare disease, there is now increasing awareness that it is underdiagnosed. ${ }^{1}$ Indeed, AATD represents a leading cause of requirement for lung and liver transplantation, ${ }^{2}$ and is the only known genetic cause of chronic obstructive pulmonary disease (COPD). ${ }^{3}$ Disease manifestations arise due to mutation in the SERPINA 1 gene located on the long arm of chromosome 14 at locus 14q332.1. ${ }^{4,5}$ This gene encodes AAT, a potent serine protease inhibitor produced primarily by hepatocytes in the liver and released into the circulation where it is the second most abundant protein. ${ }^{6}$ The primary 
role of AAT is the inhibition of proteases released from neutrophil granules. This led to the proteaseanti protease theory of emphysema which centered on AAT's role as a serine protease inhibitor with specific inhibition of neutrophil elastase (NE), an omnivorous serine protease capable of destroying almost all components of the lung matrix. The early recognition of AAT as a key inhibitor of the proteolytic enzyme NE led to the theory that emphysema in AATD was directly due to a lack of AAT. ${ }^{7}$ In support of this concept, early studies of AAT-augmentation therapy in AATD individuals demonstrated an alteration in proteolytic activity in the lungs and corresponding reduction in lung injury. ${ }^{8}$ It has since become apparent however, that the protease-antiprotease imbalance does not fully account for the pathophysiology of AATD-related disease. In this regard, predisposition of individuals with AATD to conditions characterized by aberrant neutrophilic inflammation such as panniculitis and granulomatosis with polyangiitis is suggestive of aberrant neutrophil function in the absence of standard plasma levels of AAT $(27.5 \mu \mathrm{M})$. In line with this concept, in a mouse model of AATD, AAT augmentation protected against emphysema, but oxidized AAT, that lacks anti-proteases activity, also reduced neutrophil influx into the airways. ${ }^{9}$

The most common mutation resulting in AATD is the $\mathrm{Z}$ point mutation, with substitution of a lysine for a glutamic acid residue at position 342 (Glu342Lys), leading to low levels of AAT in plasma and lung epithelial lining fluid (ELF). Moreover, Z-AAT present in the lungs has less anti-NE activity ${ }^{10}$ and is more susceptible to inhibition by oxidation, of which cigarette smoking is the most clinically relevant cause. ${ }^{11}$ Intravenous AAT augmentation therapy using pooled purified AAT at a dose of $60 \mathrm{mg} / \mathrm{kg}$ body weight is currently available for patients, with significant attenuation of decline in lung density observed with treatment. ${ }^{12,13}$

It is now recognized that AAT possesses multiple direct immuno-modulatory and anti-inflammatory activities, and strongly impacts upon the neutrophils response to a range of key cytokines and chemoattractants. ${ }^{14}$ Accordingly, increased neutrophil counts in the lungs of AATD individuals even with mild functional lung impairment, ${ }^{15}$ and also in asymptomatic, non-smoking heterozygotes for the $\mathrm{Z}$ allele or intermediate deficiency (PiMZ) without airflow obstruction 16 have been described. Moreover, studies of bronchoalveolar lavage (BAL) from nonsmoking ZZ-AATD patients showed significantly increased neutrophil numbers compared to nonsmoking AAT sufficient controls. ${ }^{17}$ While other cell types such as eosinophils and macrophages were at similar levels in both ZZ-AAT and control cohorts, ZZ-AAT patients had 3 times as many neutrophils in the absence of any infection or inflammation. ${ }^{17}$ These results formed a solid foundation for the subsequent intense studies aimed at investigating the cause of increased chemotactic activity in AATD.

\section{Targeting Neutrophil Elastase as a Driver of Neutrophil Chemokine and Cytokine Mediated Inflammation}

Initial studies exploring the ability of $\mathrm{NE}$ to induce pro-inflammatory mediates revealed that alveolar macrophages from non- smoking AATD patients, cultured in vitro, spontaneously produced higher levels of neutrophil chemotactic activity than healthy control macrophages. ${ }^{17}$ One of the most potent neutrophil chemoattractants is interleukin- 8 (IL-8). The addition of BAL obtained from people with cystic fibrosis (CF) to airway epithelial cells induced IL-8 gene expression, but in contrast, BAL obtained from healthy controls has no such effect. ${ }^{18}$ Moreover, when secretory leukoprotease inhibitor (SLPI), AAT, phenylmethanesulfonyl fluoride (PMSF) or methoxysuccinyl chlormethyl ketone, all potent inhibitors of NE, were added to CF BAL, the ability to induce IL-8 was prevented, thereby implicating NE as a major inducer of IL-8. ${ }^{19}$ These findings gave rise to the concept of a cycle of inflammation in inflammatory lung disease whereby activated neutrophils secrete NE that induces IL-8 from airway epithelium which, in turn, brings more neutrophils into the lung (Figure 1). Indeed, chemoattractants such as IL- 8 and also leukotriene B4 (LTB4) can act in a paracrine manner to further activate neutrophils, propagating their release into the inflamed airway. ${ }^{20}$ In addition to IL-8, NE induces the gene expression of a series of pro-inflammatory mediators including epithelialderived neutrophil-activating protein-78 (ENA-78), growth-regulated oncogene (GRO), interleukin-6 (IL-6), oncostatin-M (OSM), granulocyte-macrophage colony-stimulating factor (GMCSF) and vascular endothelial growth factor (VEGF) from epithelial cells. $^{21}$ Moreover, through indirect activation of 


\section{Figure 1. Cycle of Inflammation on the Respiratory Epithelial Surface in Cystic Fibrosis}

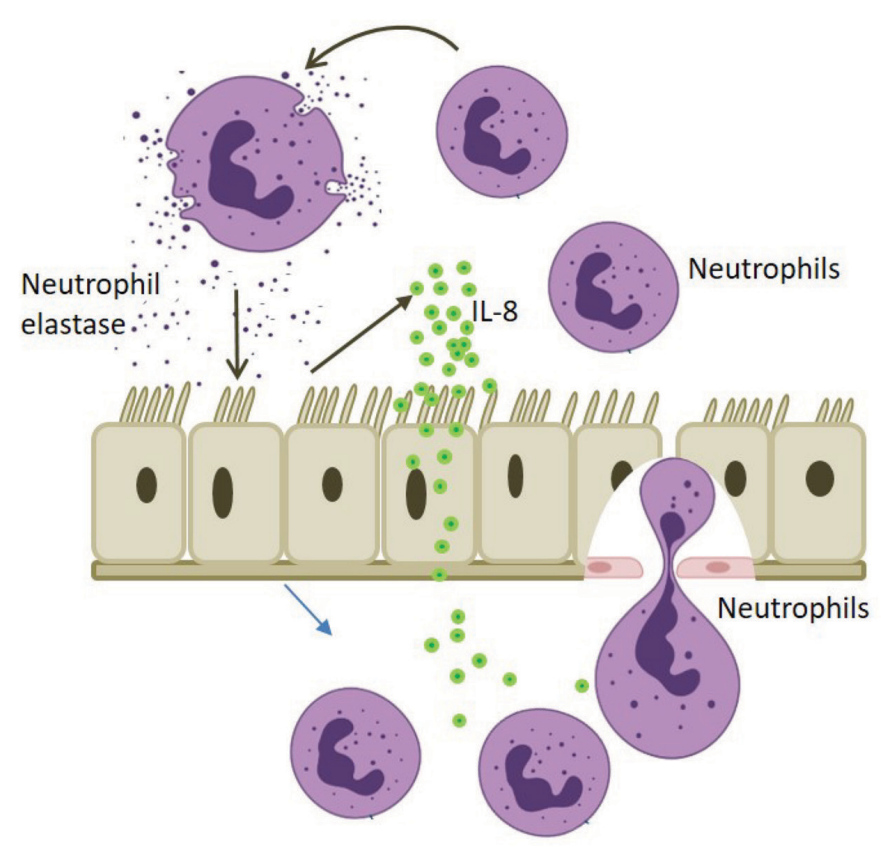

In the cystic fibrosis lung neutrophil released NE stimulates IL-8 production from airway epithelial cells. Increased IL-8 levels act as a potent chemokine drawing more neutrophils to the lungs.

$\mathrm{NE}=$ neutrophil elastase; IL-8=interleukin 8

mitogen-activated protein kinase (MAP-kinase) NE can also induce secretion of MUC5AC along with IL$8^{22}$ Inhibiting NE would therefore seem intuitively to be a good target in AATD, and indeed in vivo, NE inhibition by aerosolization of AAT or SLPI results in decreased IL-8 and airway neutrophil counts. ${ }^{18}$

Receptor signalling via NE has been intensely studied, and for example, stimulated release of IL-8from bronchial epithelial cells by $\mathrm{NE}^{23}$ can be transduced via Toll-like receptor (TLR)-4 signalling, causing de novo synthesis of the chemokine. ${ }^{24}$ Agonism of TLR4 by $\mathrm{NE}$ induces the expression of pro-inflammatory mediators downstream of nuclear factor kappalight-chain-enhancer of activated $\mathrm{B}$ cells $(\mathrm{NF}-\mathrm{kB}){ }^{25}$ The process of NE-mediated activation of the TLR4 signalling pathway may occur due to transactivation of the receptor by NE-activated protease-activated receptor-2 (PAR2) 26 or may follow cleavage of the receptor. ${ }^{27}$ Therefore, even in the absence of TLR4 on a cell, NE can elicit IL-8 production via agonism of PAR2, which in turn leads to release of ADAM metallopeptidase domain 17 from an inactive state and subsequent cleavage of transforming growth factor alpha (TGF-a). In this context, epidermal growth factor receptor (EGFR) is activated by TGF-a. ${ }^{28}$ Additionally, $\mathrm{NE}$ can directly activate the metalloprotease meprin alpha, which is capable of producing TGF-a from proTGF-a ${ }^{22}$ and can activate $\gamma \delta T$ cells to produce the pro-inflammatory effector proteins tumor necrosis factor (TNF) and interferon (IFN) $-\gamma .^{29}$

In the lung, uncontrolled NE activity yields a further number of mechanisms through which inflammation can be triggered. Digestion of certain extracellular matrix (ECM) proteins by NE, including laminin and elastin, produce characteristic peptide fragments that specifically interact with EGFR and S-gal receptor, respectively. The outcome of the former being chemoattraction of neutrophils, while the latter draws monocytes. ${ }^{30,31}$ Hence, a self-propagating cycle of immune cell recruitment and disgorgement of proteases and chemokines exists against a backdrop of ECM destruction. Moreover, excessive NE activity is not only pro-inflammatory, but antagonizes proresolving pathways. Importantly, the pro-resolving glucocorticoid, annexin A1, is inactivated following cleavage by $\mathrm{NE}$, consequently prolonging inflammatory signalling. ${ }^{32}$ The pro-resolving effect of annexin A1 can be demonstrated by therapeutic addition of the NE inhibitor SLPI to the airway. Given the extent of pathway crosstalk and temporal and contextual dependence of signalling outcomes, it seems unlikely that in vitro studies can re-capitulate the complexity of the AATD inflammatory milieu, but examination of these pathways in isolation has provided compelling reasons to therapeutically inhibit NE.

\section{Targeting Cytokines and Lipid Mediators Involved in Neutrophil Activation}

AAT is now recognized to have multiple, direct immuno-modulatory and anti-inflammatory activities, acting primarily on the neutrophil via a range of effects on key cytokines and chemo-attractants. ${ }^{14}$ Neutrophil adhesion and chemotaxis occurs in response to concentration gradients of proinflammatory lipids and cytokines including $\mathrm{LTB}_{4}$, IL-8, soluble immune complex (sIC) and also bacterial peptides including $N$-formyl-L-methionyl-L-leucyl-phenylalanine 
(fMLP). ${ }^{33}$ As a brief summary of neutrophil migration to the airways, these cells marginate to the blood vessel walls where they are tethered via interaction between ICAMs on the endothelium and selectins on the neutrophil surface, including P-selectin glycoprotein ligand 1 (PSGL-1) ${ }^{34}$ and L-selectin. ${ }^{35} \mathrm{~B}_{2}$ integrins on the neutrophil surface such as lymphocyte-associated antigen 1 (LFA 1 , integrin $\mathrm{aL} \beta_{2}, \mathrm{CD} 11 \mathrm{a} / \mathrm{CD} 18$ ) and macrophage- 1 antigen (Mac-1, integrin $\mathrm{aM} \beta_{2}, \mathrm{CD} 11 \mathrm{~b} /$ CD18) interact with ICAMs on the endothelium, resulting in firm adhesion and crawling of neutrophils along the vessel wall to reach junctions between endothelial cells. ${ }^{36,37}$ Interaction between neutrophil $\beta_{2}$ integrins and endothelial ICAMs and junctional adhesion molecules then mediates transmigration of the cell through the endothelial junction. ${ }^{38}$ Matrix metalloproteases released from neutrophil gelatinase granules digest the basement membrane protein and neutrophils enter the interstitial space. ${ }^{39}$ AATD individuals, regardless of smoking status, demonstrate increased neutrophil chemotactic index, ${ }^{17}$ strongly indicating that AAT affects neutrophil migratory patterns. AAT may exert this anti-inflammatory effect by 1 of 2 ways; the first involving down-regulation of pro-inflammatory cytokines and chemokine protein production, the second concerning modulation of downstream signalling events. In agreement with the former suggestion, it has been shown that NE induces IL-8 gene up-regulation in bronchial epithelial cells through an IRAK signalling pathway involving both MyD88 and TRAF-6, and that the serine protease inhibitors SLPI and AAT, prevent IL-8 gene activation and protein production. ${ }^{19}$ Of interest, glycosaminoglycans influence the chemokine profile of the CF lung by binding IL- 8 and protecting it from proteolytic degradation; this binding event offers therapeutic opportunities. For example, the IL-8 decoy molecule PA401 which lacks chemotactic activity, was shown to disrupt IL-8: glycosaminoglycan complexes present in CF airway samples, rendering IL-8 susceptible to NE proteolysis and clearance. ${ }^{40}$ Consequently, this decoy approach represents a novel approach to targeting high levels of IL- 8 and may serve to decrease the inflammatory burden in the AATD lung.

The alternative method by which the antiprotease AAT may modulate neutrophil adhesion and chemotaxis is by binding to, and blocking, either ligand or receptor. An example of this is the immune- regulatory effect of AAT on $\mathrm{LTB}_{4}$. This lipid mediator is a potent inducer of neutrophil adhesion via its receptor BLT1. LTB 4 is secreted from both macrophages and neutrophils upon exposure to $\mathrm{NE}^{17,41}$ thereby drawing further neutrophils to the airways. Breaking this cycle of inflammation is of importance, and AAT was shown to modulate neutrophil adhesion via AAT-LTB 4 complex formation, consequently inhibiting $\mathrm{LTB}_{4}$ BLT1 interaction. ${ }^{41}$ The latter study identified the hydrophobic pocket on AAT located between S2A and helices $D$ and $E$ as the potential binding site for $\mathrm{LTB}_{4}$. Of note, AAT did not cause complete inhibition of LTB4-BLT 1 engagement and this is a further important attribute of AAT as an $\mathrm{LTB}_{4}$ antagonist. Moreover, results of this study revealed reduced plasma levels of $\mathrm{LTB}_{4}$ in AATD patients receiving AAT augmentation therapy, compared with untreated patients matched by forced expiratory volume in 1 second. ${ }^{41}$

Progressing from adhesion to chemotaxis, SLPI has been shown to significantly inhibit fMLP and IL-8 induced neutrophil chemotaxis, with lower levels of cytosolic inositol 1,4,5-triphosphate and calcium flux detected. ${ }^{42}$ Although the exact mechanism by which SLPI blocks fMLP/IL-8 cell responses is not clear, extensive research has been carried out on the ability of AAT to inhibit IL-8 induced neutrophil chemotaxis. ${ }^{43}$ Results have demonstrated the ability of glycosylated AAT to bind IL-8 via an electrostatic interaction. IL-8 bound to AAT is blocked from interacting with CXCR1, thus preventing actin cytoskeletal rearrangements and intra-cellular calcium flux required for the chemotaxis process. In line with this concept, in vivo, alterations of AAT glycan signatures involving increased levels of sialylation during the time course of acute infection and resolution of community-acquired pneumonia, reduced IL-8 binding to neutrophil CXCR1. ${ }^{44}$ Moreover, AAT has been shown to bind directly to circulating neutrophils, localized to membrane lipid rafts, and inhibiting ADAM-17. ADAM-17 cleaves the glycosylphosphatidylinositol-anchored Fc receptor FcyRIIIb (CD16b) from the cell surface, a process which is crucial for chemotaxis in response to sIC. Inhibition of ADAM-17 by AAT prevents release of CD16b, thereby downregulating chemotaxis in response to sIC. This study demonstrated increased neutrophil chemotaxis in AATD individuals in response to IL-8 and SIC, which normalized following AAT augmentation therapy in vivo. ${ }^{43}$

TNF-a is an important pro-inflammatory cytokine 
that modulates neutrophil degranulation of secondary and tertiary granules. ${ }^{45}$ Secondary granules are a source of lactoferrin and hCAP-18, whilst tertiary granules contain matrix-metalloprotease-9 (MMP-9). Neutrophils of AATD individuals display increased production and membrane expression of TNF-a compared to healthy control cells, and release elevated levels of secondary and tertiary granule components. ${ }^{46}$ In this latter study, extracellular supernatants from TNF-a stimulated AATD neutrophils contained higher concentrations of MMP-9, hCAP-18 and lactoferrin compared to healthy controls, indicating augmented secondary and tertiary granule release. Furthermore, excessive degranulation of lactoferrin resulted in an increased presence of IgG class anti-lactoferrin antibodies which consequently augmented production of reactive oxygen species, suggesting a role for autoimmunity in the pathophysiology of AATD. In vitro, exogenous AAT was shown to bind to TNF-a receptors (TNFR1 and TNFR2), preventing activation of the MAPK p38 phosphorylation pathway. ${ }^{47}$ Of major importance, TNF-a signalling possesses the ability to self-regulate its own gene expression and in this latter study, AAT down-regulated TNF-a gene expression in response to exogenous TNF-a and caused a blockade of $\mathrm{IkBa}$ degradation, thereby preventing induction of NF-kB-regulated pro-inflammatory mediators. Moreover, in vivo in AATD patients, AAT augmentation therapy reduced soluble plasma levels of TNFR1, a surrogate marker for TNF-a. ${ }^{46}$

Collectively, this published data demonstrates that AATD is associated with dysregulated neutrophil function, with increased chemotaxis, adhesion and degranulation. The consequences of this are increased neutrophil burden in the lung, compounded by increased release of granule proteins including $\mathrm{NE}$ and perseverance of inflammation. A variety of AAT cytokine and lipid immunoregulatory mechanisms have been implicated, including direct interaction between AAT and IL-8 as well as LTB down-regulation of TNF-a pathways by AAT, autoimmunity, and effects of excess NE in stimulating IL-8 production. Importantly, exogenous AAT in vivo has been shown to correct many of these events, resulting in normalization of chemotaxis ${ }^{43}$ adhesion and degranulation. ${ }^{41}$ The effects of AAT augmentation therapy on neutrophilic inflammation are thus worthy of further investigation.

\section{Interleukin-1 $\beta$ as a Target for Immunomodulation in Alpha-1 Antitrypsin Deficiency}

A pro-inflammatory mediator currently of major interest is interleukin-1 $\beta$ (IL-1 $\beta$ ), due to its induction in numerous cell types under strong inflammatory stimulation, and ability to in turn stimulate a broad array of pro-inflammatory responses. Release of IL-1 $\beta$ begins with synthesis of proIL- $1 \beta$ that is cleaved by intracellular caspase- 1 activity pursuant to assembly of an inflammasome complex. The predominant complex driving IL-1 $\beta$ production is the NLRP3 inflammasome and, notably, NLRP3 deficient mice fail to match their normal counterparts in development of a COPD phenotype. ${ }^{48}$ Neither NLRP3 nor IL-1 $\beta$ itself are detectably elevated in COPD lung tissue, ${ }^{49}$ though levels of IL- $1 \beta$ are higher in both the serum and BALF of those with COPD than smokers with otherwise normal spirometry, suggesting that substantial inflammatory signalling is required to elicit the cytokine. ${ }^{50}$ This may include apoptosis or even necrosis-associated danger signals or infectious exacerbations. ${ }^{51}$ The latter observation is telling given the dominant influence of the TLR4 ligand lipopolysaccharide (LPS) on NLRP3 inflammasome activation. Moreover, while anti-IL-1 $\beta$ therapy is minimally effective in stable COPD patients ${ }^{52,53}$ - the effective absence of AAT in deficient individuals may be decisive in establishing a role for IL-1 $\beta$ in AATD.

Insofar as the NLRP3 (NLR Family Pyrin Domain Containing 3) inflammasome is a sensor of cellular disequilibrium, it can become activated during osmotic stress such as sodium influx. ${ }^{54}$ This effect can be mediated through the epithelial sodium channel (ENaC). Normally, $\mathrm{ENaC}$ is minimally active due to regulation by extracellular SPLUNC1 (short palate lung and nasal epithelial clone 1). ${ }^{55}$ Diminished AAT has been shown to coincide with reduced levels of SPLUNC1 ${ }^{56}$ suggesting that heightened ENaC activity and, hence, active NLRP3 may occur in AATD. Moreover, AAT can further protect tissues from IL$1 \beta$ signalling by up-regulating interluekin-1 receptor antagonist (IL-1Ra), either directly from the tissue or secreted by macrophages or regulatory $\mathrm{T}$ cells. ${ }^{57,58}$ Hence, in AATD, this normally protective mechanism could be diminished and levels of IL-1 $\beta$ would be expectedly elevated. In line with this thought, AAT can suppress the ability of extracellular ATP to induce 
proIL-1 $\beta$ expression and this action of AAT occurs independently of its anti-NE function. ${ }^{59}$ AAT may achieve this control of IL- $1 \beta$ maturation by activation of phospholipase A2, which in turn prevents ATP receptor P2X7 signalling. The source of extracellular ATP may coincide with the release of IL-1 $\beta$ from the cell given that both require membrane permeability. ${ }^{60}$ Concordantly, AAT can inhibit the inducer of apoptosis, caspase-3, thereby restricting IL-1 $\beta$ release. This inhibitory action of AAT occurs directly, requiring the native reactive center loop of AAT, and following internalization of the anti-protease, ${ }^{61}$ which likely occurs through clathrin-mediated endocytosis. ${ }^{62}$ Membrane permeability is also a consequence of pore formation by gasdermin $D$, causing a form of cell death termed pyroptosis, which is instigated by inflammasome activity. Release of IL-1 $\beta$ as well as ATP and other danger signals then follows. As such, IL-1 $\beta$ release may be self-propagating, thereby amplifying inflammation once the initiating triggers are present.

Diminished oxygenation is another route through which inflammasomes can become activated, whereby the glycolytic metabolite lactate promotes stabilization of HIF $1 \mathrm{a}$ and subsequent expression of pro-inflammatory genes, including proIL-1 $\beta$. Hence, in severely obstructed airways, IL-1 $\beta$ may be pronounced. Notably, glycolysis may also occur in normoxic conditions, in the presence of Gramnegative bacteria. Here, LPS ligation by TLR4 triggers the NLRP3 inflammasome-directed maturation of IL$1 \beta$. This effect was recently demonstrated for the first time to occur in neutrophils of patients with cystic fibrosis. ${ }^{63}$ Hence, conditions in the AATD airway can replicate those required for IL-1 $\beta$ production and processing by neutrophils: pro-inflammatory cytokine levels are above normal and many individuals are colonized by Gram- negative bacteria, such as Haemophilus influenzae or Moraxella catharrhalis. The "hyperactivation" of the neutrophil in AATD, the result of excessive neutrophil serine protease activity and abundant neutrophil chemoattractants, gives the strong probability that inhibitors of NLRP3 activation may mitigate the inflammatory burden in the AATD airway. Of caution, it should be noted that therapeutic intervention against IL-1 $\beta$ can result in increased incidence of infection as can occur when IL-1 signalling is blocked by recombinant IL-1Ra, anakinra. ${ }^{64}$ This adverse outcome is likely the result of insufficient pro-inflammatory, anti-infective immunity. Hence, the effect of anti-IL-1 $\beta$ therapy on infective exacerbations in AATD should be considered carefully. That said, where an exacerbation is already in progress, anti-IL$1 \beta$ therapy may show benefit in controlling an overly exuberant immune response, thereby shortening time to resolution. Additionally, more precise modulation of the immune response may be achieved instead by inhibiting NLRP3 activity, ${ }^{63}$ thereby preserving IL-1a signalling.

\section{Conclusion}

Our understanding of AATD has evolved from a purely protease-driven pathogenesis to an appreciation of the central role of inflammation in the condition. Much of this has stemmed from better understanding of the many faceted actions of AAT. As befits an abundant plasma protein, AAT has systemic anti-inflammatory effects as well as local actions in the lung. There is significant crosstalk between proteases and inflammatory pathways. Some of the anti-inflammatory effects of AAT certainly derive from inhibiting NE with subsequent downstream consequences, such as decreased production of neutrophil chemokines and reduced activation of further proteases (Table 1). The non-anti-protease effects of AAT differ according to the inflammatory process involved and also, structural characteristics of the AAT protein. The anti-IL-8 activity of AAT requires AAT glycosylation, and the anti-LTB 4 effect utilizes a hydrophobic domain, while the anti-TNF effects do not directly affect TNF but rather block its interaction with its receptors. What is clear is that AATD is characterized not only by unopposed protease activity, but a lack of AAT antiinflammatory effects and that cytokine signalling including IL-1 $\beta$ should be considered equally critical (Figure 2).

\section{Declaration of Interest}

All authors have no conflict of interests to declare 


\section{Table 1. Alpha-1 Antitrypsin Deficiency With Associated Inflammatory Mediators and Triggers That May Contribute to Disease}

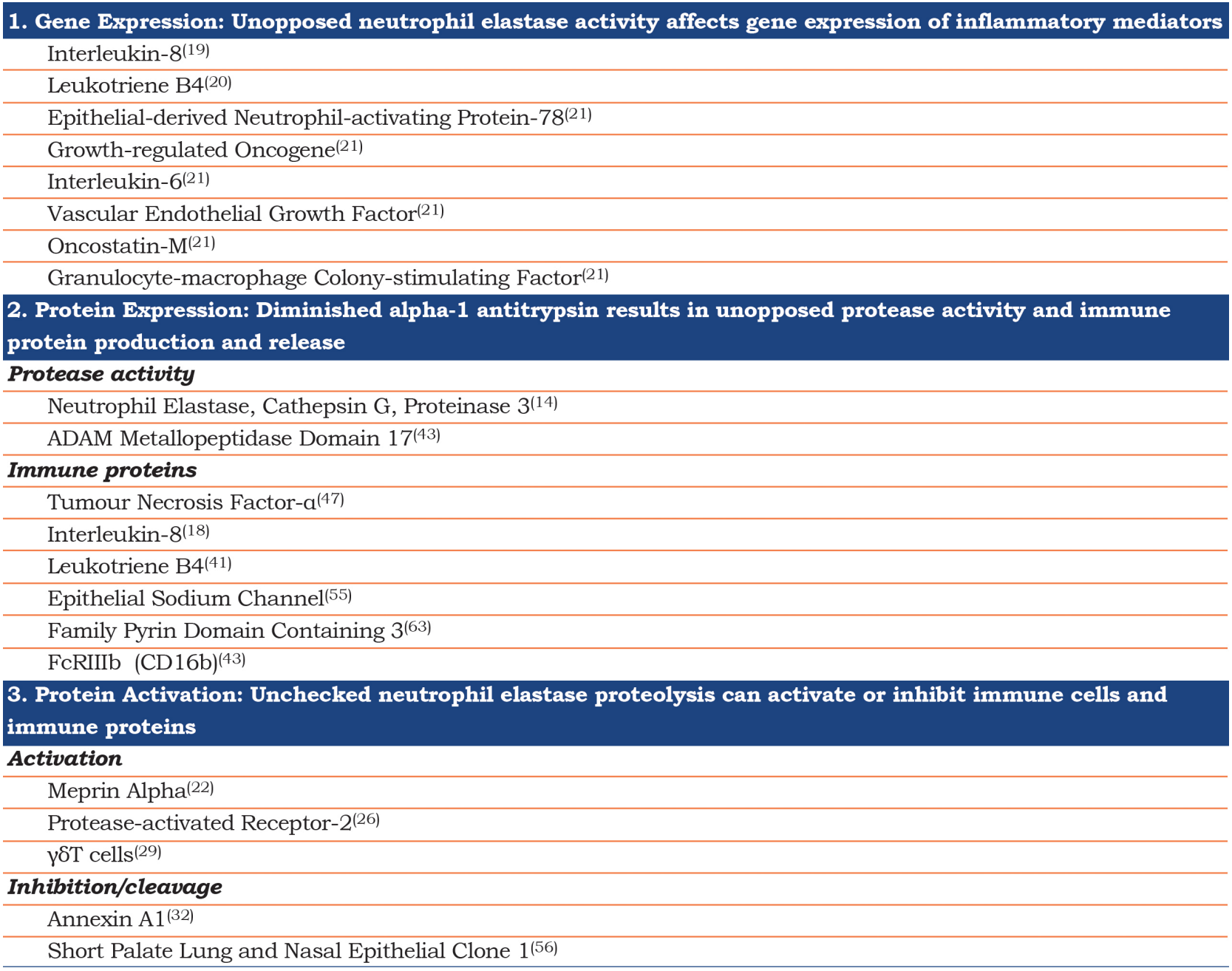




\section{Figure 2. Alpha-1 Antitrypsin Deficiency Cells Are Primed for Pro-inflammatory Signalling}

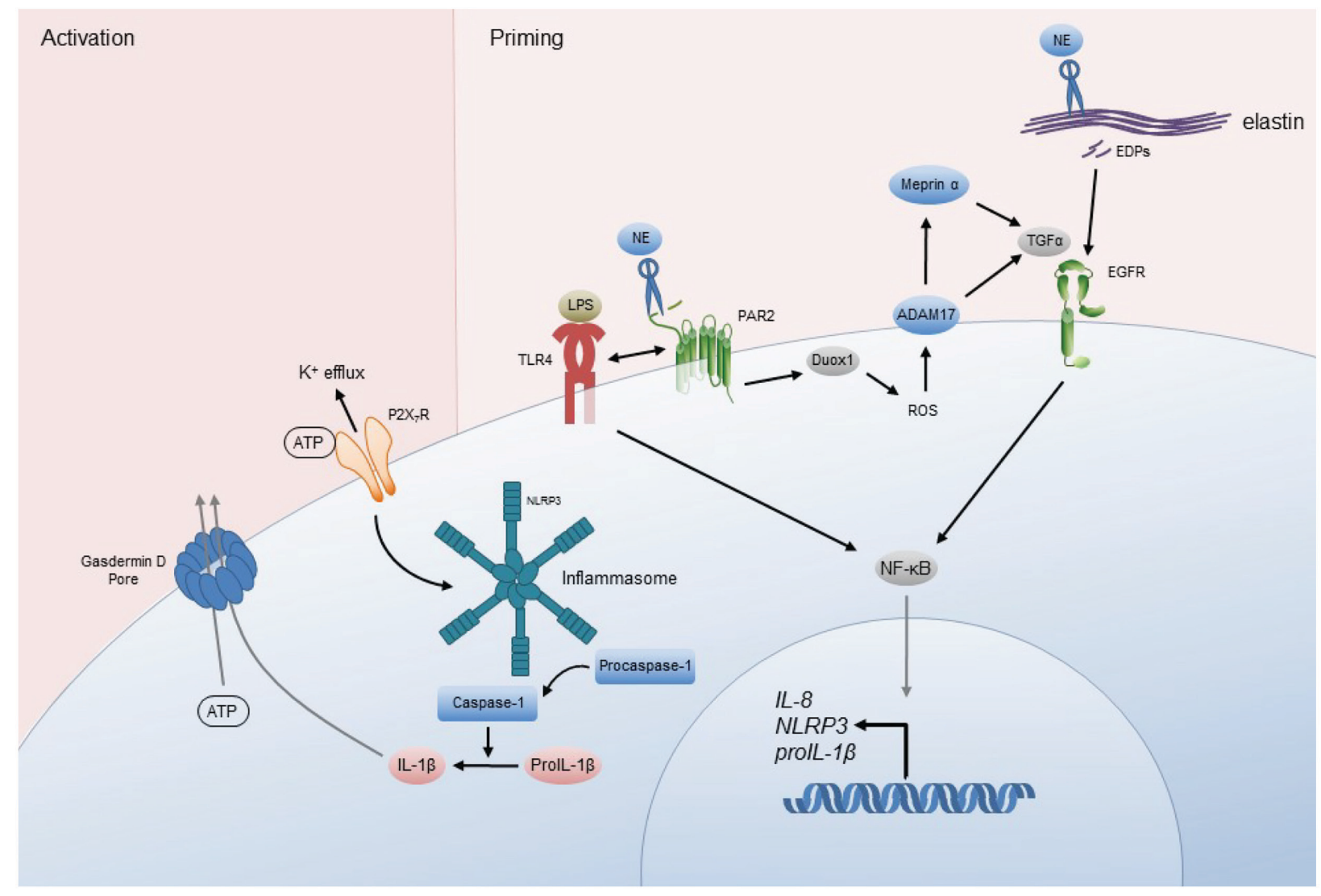

Where AAT is insufficient, the action of NE may serve to prime cells such that inflammasome assembly is activated in response to pro-inflammatory stimuli. NE activity signals through TLRs, PAR2 or EGFR, which induces the expression of pro-inflammatory genes that are regulated by NF-kB. Hence, NLRP3 and proIL-1 $\beta$ are available for the production and release of active IL-1 $\beta$, alongside other pro-inflammatory mediators including chemoattractants and endogenous danger signals.

$\mathrm{AAT}=$ alpha-1 antitrypsin; $\mathrm{NE}=$ neutrophil elastase; TLRs=toll-like receptors; PAR2=protease-activated receptor 2; EGFR=epidermal growth factor receptor; NF-kB=nuclear factor kappa-light-chain-enhancer of activated B cells; NLR3=Family Pyrin Domain Containing 3; IL- $1 \beta=$ interleukin $1 \beta$ 


\section{References}

1. McElvaney NG. Diagnosing alpha-1 antitrypsin deficiency: how to improve the current algorithm. Eur Respir Rev. 2015;24(135):52-57.

doi: https://doi.org/10.1183/09059180.10010814

2. Stoller JK, Brantly M. The challenge of detecting alpha-1 antitrypsin deficiency. COPD. 2013;10(Suppl 1):26-34. doi: https://doi.org/10.3109/15412555.2013.763782

3. Vogelmeier CF, Criner GJ, Martinez FJ, et al. Global strategy for the diagnosis, management, and prevention of chronic obstructive lung disease 2017 Report: GOLD executive summary. Eur Respir J. 2017;49(3). doi: https://doi.org/10.1183/13993003.00214-2017

4. Darlington GJ, Astrin KH, Muirhead SP, Desnick RJ, Smith M. Assignment of human alpha- 1 antitrypsin to chromosome 14 by somatic cell hybrid analysis. Proc Natl Acad Sci U S A. 1982;79(3):870-873. doi: https://doi.org/10.1073/pnas.79.3.870

5. Cox DW, Markovic VD, Teshima IE. Genes for immunoglobulin heavy chains and for alpha- 1 antitrypsin are localized to specific regions of chromosome 14q. Nature. 1982;297(5865):428-430. doi: https://doi.org/10.1038/297428a0

6. Huber R, Carrell RW. Implications of the three-dimensional structure of alpha-1 antitrypsin for structure and function of serpins. Biochemistry.1989;28(23):8951-8966. doi: https://doi.org/10.1021/bi00449a001

7. Gadek JE, Fells GA, Zimmerman RL, Rennard SI, Crystal RG. Antielastases of the human alveolar structures. Implications for the protease-antiprotease theory of emphysema. J Clin Invest. 1981;68(4):889-898. doi: https://doi.org/10.1172/JCI110344

8. Wewers MD, Casolaro MA, Sellers SE, et al. Replacement therapy for alpha-1 antitrypsin deficiency associated with emphysema. N Engl J Med. 1987;316(17):1055-1062.

doi: https://doi.org/10.1056/NEJM198704233161704

9. Churg A, Wang RD, Xie C, Wright JL. Alpha-1 antitrypsin ameliorates cigarette smoke-induced emphysema in the mouse. Am J Respir Crit Care Med. 2003;168(2):199-207. doi: https://doi.org/10.1164/rccm.200302-203OC

10. Ogushi F, Fells GA, Hubbard RC, Straus SD, Crystal RG. Z-type alpha-1 antitrypsin is less competent than M1-type alpha-1 antitrypsin as an inhibitor of neutrophil elastase. J Clin Invest. 1987;80(5):1366-1374. doi: https://doi.org/10.1172/JCI113214

11. Gadek JE, Fells GA, Crystal RG. Cigarette smoking induces functional antiprotease deficiency in the lower respiratory tract of humans. Science. 1979;206(4424):1315-1316.

doi: https://doi.org/10.1126/science.316188
12. Chapman KR, Burdon JG, Piitulainen E, et al. Intravenous augmentation treatment and lung density in severe alpha-1 antitrypsin deficiency (RAPID): a randomised, double-blind, placebo-controlled trial. Lancet. 2015;386(9991):360-368. doi: https://doi.org/10.1016/S0140-6736(15)60860-1

13. McElvaney NG, Burdon J, Holmes M, et al. Long-term efficacy and safety of alpha-1 proteinase inhibitor treatment for emphysema caused by severe alpha-1 antitrypsin deficiency: an open-label extension trial (RAPID-OLE). Lancet Respir Med. 2017;5(1):5160. doi: https://doi.org/10.1016/S2213-2600(16)30430-1

14. Bergin DA, Hurley K, McElvaney NG, Reeves EP. Alpha-1 antitrypsin: a potent anti-inflammatory and potential novel therapeutic agent. Arch Immunol Ther Exp (Warsz). 2012;60(2):81-97.

doi: https://doi.org/10.1007/s00005-012-0162-5

15. Rouhani F, Paone G, Smith NK, Krein P, Barnes P, Brantly ML. Lung neutrophil burden correlates with increased pro-inflammatory cytokines and decreased lung function in individuals with alpha 1-antitrypsin deficiency. Chest. 2000;117(5 Suppl 1):250S-1S. doi: https://doi.org/10.1378/chest.117.5_suppl_1.250S

16. Malerba M, Ricciardolo F, Radaeli A, et al. Neutrophilic inflammation and IL-8 levels in induced sputum of alpha-1antitrypsin PiMZ subjects. Thorax. 2006;61(2):129-133. doi: https://doi.org/10.1136/thx.2005.043471

17. Hubbard RC, Fells G, Gadek J, Pacholok S, Humes J, Crystal RG. Neutrophil accumulation in the lung in alpha- 1 antitrypsin deficiency. Spontaneous release of leukotriene B4 by alveolar macrophages. J Clin Invest. 1991;88(3):891-897. doi: https://doi.org/10.1172/JCI115391

18. McElvaney NG, Nakamura H, Birrer P, Hebert CA, et al. Modulation of airway inflammation in cystic fibrosis. In vivo suppression of interleukin-8 levels on the respiratory epithelial surface by aerosolization of recombinant secretory leukoprotease inhibitor. J Clin Invest. 1992;90(4):1296-1301. doi: https://doi.org/10.1172/JCI115994

19. Walsh DE, Greene CM, Carroll TP, et al. Interleukin-8 upregulation by neutrophil elastase is mediated by MyD88/ IRAK/TRAF-6 in human bronchial epithelium. $J$ Biol Chem. 2001;276(38):35494-35499.

doi: https://doi.org/10.1074/jbc.M103543200

20. Serezani CH, Lewis C, Jancar S, Peters-Golden M. Leukotriene B4 amplifies NF-kappaB activation in mouse macrophages by reducing SOCS 1 inhibition of MyD88 expression. J Clin Invest. 2011;121(2):671-682. doi: https://doi.org/10.1172/JCI43302

21. Carroll TP, Greene CM, Taggart CC, Bowie AG, O’Neill SJ, McElvaney NG. Viral inhibition of IL-1- and neutrophil elastaseinduced inflammatory responses in bronchial epithelial cells. $J$ Immunol. 2005; 175(11):7594-7601. doi: https://doi.org/10.4049/jimmunol.175.11.7594 
22. Bergin DA, Greene CM, Sterchi EE, et al. Activation of EGFR by a novel metalloprotease pathway. J Biol Chem. 2008;283:3173831744. doi: https://doi.org/10.1074/jbc.M803732200

23. Walsh DE, Greene CM, Carroll TP, et al. Interleukin-8 upregulation by neutrophil elastase is mediated by MyD88/ IRAK/TRAF-6 in human bronchial epithelium. J Biol Chem. 2001;276(38):35494-35499.

doi: https://doi.org/10.1074/jbc.M103543200

24. Devaney JM, Greene CM, Taggart CC, Carroll TP, O’Neill SJ, McElvaney NG. Neutrophil elastase up-regulates interleukin-8 via toll-like receptor 4. FEBS Lett. 2003;544(1-3):129-132. doi: https://doi.org/10.1016/S0014-5793(03)00482-4

25. Carroll TP, Greene CM, Taggart CC, Bowie AG, O’Neill SJ, McElvaney NG. Viral inhibition of IL-1- and neutrophil elastaseinduced inflammatory tesponses in bronchial epithelial cells. $J$ Immunol. 2005;175(11):7594-7601.

doi: https://doi.org/10.4049/jimmunol.175.11.7594

26. Yamaguchi R, Yamamoto T, Sakamoto A, et al. A proteaseactivated receptor 2 agonist (AC-264613) suppresses interferon regulatory factor 5 and decreases interleukin-12p 40 production by lipopolysaccharide-stimulated macrophages: role of p53. Cell Biol Int. 2016;40(6):629-41.

doi: https://doi.org/10.1002/cbin.10589

27. Benabid R, Wartelle J, Malleret L, Guyot N, Gangloff S, Lebargy F, et al. Neutrophil elastase modulates cytokine expression: contribution to host defense against pseudomonas aeruginosainduced pneumonia. J Biol Chem. 2012;287(42):34883-34894. doi: https://doi.org/10.1074/jbc.M112.361352

28. Kuwahara I, Lillehoj EP, Lu W, et al. Neutrophil elastase induces IL-8 gene transcription and protein release through p38/NF$\mathrm{kB}$ activation via EGFR transactivation in a lung epithelial cell line. Am J Physiol Lung Cell Mol Physiol. 2006;291(3):L407-L16. doi: https://doi.org/10.1152/ajplung.00471.2005

29. Towstyka NY, Shiromizu CM, Keitelman I, et al. Modulation of gammadelta T-cell activation by neutrophil elastase. Immunology. 2018;153(2):225-237.

doi: https://doi.org/10.1111/imm.12835

30. Mydel P, Shipley JM, Adair-Kirk TL, et al. Neutrophil elastase cleaves laminin-332 (laminin-5) generating peptides that are chemotactic for neutrophils. J Biol Chem. 2008;283(15):95139522. doi: https://doi.org/10.1074/jbc.M706239200

31. Hunninghake GW, Davidson JM, Rennard S, Szapiel S, Gadek JE, Crystal RG. Elastin fragments attract macrophage precursors to diseased sites in pulmonary emphysema. Science. 1981;212(4497):925-927.

doi: https://doi.org/10.1126/science.7233186

32. Vago JP, Tavares LP, Sugimoto MA, et al. proresolving actions of synthetic and natural protease inhibitors are mediated by annexin A1. J Immunology. 2016;196(4):1922-1932. doi: https://doi.org/10.4049/jimmunol.1500886
33. Borregaard N. Neutrophils from marrow to microbes. Immunity. 2010;33(5):657-670.

doi: https://doi.org/10.1016/j.immuni.2010.11.011

34. McEver RP, Cummings RD. Role of PSGL-1 binding to selectins in leukocyte recruitment. J Clin Invest. 1997;100(11 Suppl):S97-103. doi: https://doi.org/10.1172/JCI119556

35. Kansas GS. Selectins and their ligands: current concepts and controversies. Blood. 1996;88(9):3259-3287. doi: https://doi. org/10.1182/blood.V88.9.3259.bloodjournal8893259

36. Massena S, Christoffersson G, Hjertstrom E, et al. A chemotactic gradient sequestered on endothelial heparan sulfate induces directional intraluminal crawling of neutrophils. Blood. 2010;116(11): 1924-1931.

doi: https://doi.org/10.1182/blood-2010-01-266072

37. Summers C, Rankin SM, Condliffe AM, Singh N, Peters AM, Chilvers ER. Neutrophil kinetics in health and disease. Trends Immunol. 2010;31(8):318-324. doi: https://doi.org/10.1016/j.it.2010.05.006

38. Ley K, Laudanna C, Cybulsky MI, Nourshargh S. Getting to the site of inflammation: the leukocyte adhesion cascade updated. Nat Rev Immunol. 2007;7(9):678-689.

doi: https://doi.org/10.1038/nri2156

39. Delclaux C, Delacourt C, D'Ortho MP, Boyer V, Lafuma C, Harf A. Role of gelatinase $\mathrm{B}$ and elastase in human polymorphonuclear neutrophil migration across basement membrane. Am J Respir Cell Mol Biol. 1996;14(3):288-95.

doi: https://doi.org/10.1165/ajrcmb.14.3.8845180

40. McElvaney OJ, O'Reilly N, White M, et al. The effect of the decoy molecule PA401 on CXCL8 levels in bronchoalveolar lavage fluid of patients with cystic fibrosis. Mol Immunol. 2015;63(2):550-558. doi: https://doi.org/10.1016/j.molimm.2014.10.013

41. O'Dwyer CA, O'Brien ME, Wormald MR, et al. The BLT1 inhibitory function of alpha-1 antitrypsin augmentation therapy disrupts leukotriene B4 neutrophil signaling. J Immunol. 2015; 195(8):3628-3641.

doi: https://doi.org/10.4049/jimmunol.1500038

42. Reeves EP, Banville N, Ryan DM, et al. Intracellular secretory leukoprotease inhibitor modulates inositol 1,4,5-triphosphate generation and exerts an anti-inflammatory effect on neutrophils of individuals with cystic fibrosis and chronic obstructive pulmonary disease. Biomed Res Int. 2013;2013:560141. doi: https://doi.org/10.1155/2013/560141

43. Bergin DA, Reeves EP, Meleady P, et al. Alpha-1 antitrypsin regulates human neutrophil chemotaxis induced by soluble immune complexes and IL-8. J Clin Invest. 2010;120(12):42364250. doi: https://doi.org/10.1172/JCI41196

44. McCarthy C, Dimitrov BD. Augmentation therapy for alpha-1 antitrypsin deficiency-not enough evidence to support its use yet! COPD. 2010;7(3):234-235.

doi: https://doi.org/10.3109/15412555.2010.485108 
45. von Haehling S, Hopkinson NS, Polkey MI, Niethammer M, Anker SD, Genth-Zotz S. Elevated TNFalpha production in whole blood in patients with severe COPD: the potential link to disease severity. Wien Klin Wochenschr. 2009;121(9-10):303-308. doi: https://doi.org/10.1007/s00508-009-1186-7

46. Bergin DA, Reeves EP, Hurley K, et al. The circulating proteinase inhibitor alpha-1 antitrypsin regulates neutrophil degranulation and autoimmunity. Sci Transl Med. 2014;6(217):217ra1. doi: https://doi.org/10.1126/scitranslmed.3007116

47. Mocsai A, Jakus Z, Vantus T, Berton G, Lowell CA, Ligeti E. Kinase pathways in chemoattractant-induced degranulation of neutrophils: the role of p38 mitogen-activated protein kinase activated by SRC family kinases. J Immunol. 2000;164(8):43214331. doi: https://doi.org/10.4049/jimmunol.164.8.4321

48. Yang W, Ni H, Wang H, Gu H. NLRP3 inflammasome is essential for the development of chronic obstructive pulmonary disease. Int J Clin Exp Pathol. 2015;8(10):13209-13216.

49. Di Stefano A, Caramori G, Barczyk A, Vicari C, Brun P, Zanini A, et al. Innate immunity but not NLRP3 inflammasome activation correlates with severity of stable COPD. Thorax. 2014;69(6):516524. doi: https://doi.org/10.1136/thoraxjnl-2012-203062

50. Wang $\mathrm{H}$, Lv Ce, Wang S, Ying $\mathrm{H}$, Weng $\mathrm{Y}$, Yu W. NLRP3 Inflammasome involves in the acute exacerbation of patients with chronic obstructive pulmonary disease. Inflammation. 2018;41(4):1321-1333.

doi: https://doi.org/10.1007/s 10753-018-0780-0

51. Faner R, Sobradillo P, Noguera A, et al. The inflammasome pathway in stable COPD and acute exacerbations. ERJ Open Research. 2016;2(3):00002-2016. doi: https://doi.org/10.1183/23120541.00002-2016

52. Calverley PMA, Sethi S, Dawson M, et al. A randomised, placebocontrolled trial of anti-interleukin-1 receptor 1 monoclonal antibody MEDI8968 in chronic obstructive pulmonary disease. Respir Res. 2017;18(1):153.

doi: https://doi.org/10.1186/s12931-017-0633-7

53. National Institutes of Health. Safety and efficacy of multiple doses of canakinumab (acz885) in chronic obstructive pulmonary disease (COPD) patients. NCT00581945. Published: December 28, 2007. Updated: June 30, 2011. Accessed: October 16, 2019. https:/clinicaltrials.gov/ct2/show/NCT00581945

54. Scambler T, Jarosz-Griffiths HH, Lara-Reyna S, et al. ENaCmediated sodium influx exacerbates NLRP3-dependent inflammation in cystic fibrosis. eLife. 2019;8:e49248.

doi: https://doi.org/10.7554/eLife.49248

55. Kim CS, Ahmad S, Wu T, Walton WG, Redinbo MR, Tarran R. SPLUNC1 is an allosteric modulator of the epithelial sodium channel. FASEB J. 2018;32(5):2478-2491.

doi: https://doi.org/10.1096/f.201701126R
56. Jiang D, Persinger R, Wu Q, Gross A, Chu HW. a1-antitrypsin promotes SPLUNC1-mediated lung defense against Pseudomonas aeruginosa infection in mice. Respir Res. 2013;14(1):122. doi: https://doi.org/10.1186/1465-9921-14-122

57. Abecassis A, Schuster R, Shahaf G, et al. a1-antitrypsin increases interleukin-1 receptor antagonist production during pancreatic islet graft transplantation. Cell Mol Immunol. 2014;11(4):377386. doi: https://doi.org/10.1038/cmi.2014.17

58. Lewis EC, Mizrahi M, Toledano M, et al. a1-antitrypsin monotherapy induces immune tolerance during islet allograft transplantation in mice. Proc Natl Acad Sci U S A. 2008; 105(42):16236-16241.

doi: https://doi.org/10.1073/pnas.0807627105

59. Siebers K, Fink B, Zakrzewicz A, et al. Alpha-1 antitrypsin inhibits ATP-mediated release of interleukin-1 $\beta$ via CD36 and nicotinic acetylcholine receptors. Front Immunol. 2018;9:877. doi: https://doi.org/10.3389/fimmu.2018.00877

60. Tapia VS, Daniels MJD, Palazon-Riquelme P, et al. The three cytokines IL-1beta, IL-18, and IL-1alpha share related but distinct secretory routes. J Biol Chem. 2019;294(21):8325-8335. doi: https://doi.org/10.1074/jbc.RA119.008009

61. Petrache I, Fijalkowska I, Medler TR, et al. Alpha-1 antitrypsin inhibits caspase- 3 activity, preventing lung endothelial cell apoptosis. Am J Pathol. 2006;169(4):1155-1166. doi: https://doi.org/10.2353/ajpath.2006.060058

62. Wang J, Gou W, Kim DS, Strange C, Wang H. Clathrin-mediated endocytosis of alpha-1 antitrypsin is essential for its protective function in islet cell survival. Theranostics. 2019;9(13):39403951. doi: https://doi.org/10.7150/thno.31647

63. McElvaney OJ, Zaslona Z, Becker-Flegler K, et al. Specific inhibition of the NLRP3 inflammasome as an anti-inflammatory strategy in cystic fibrosis. Am J Respir Crit Care Med. 2019. doi: https://doi.org/10.1164/rccm.201905-10130C

64. Salliot C, Dougados M, Gossec L. Risk of serious infections during rituximab, abatacept and anakinra treatments for rheumatoid arthritis: meta-analyses of randomised placebocontrolled trials. Ann Rheum Dis. 2009;68(1):25-32. doi: https://doi.org/10.1136/ard.2007.083188 